\title{
Weldability of a High Entropy CrMnFeCoNi Alloy
}

\author{
Z. Wu, S. A. David, Z. Feng, H. Bei *
}

Materials Science and Technology Division, Oak Ridge National Laboratory, Oak Ridge, TN 37831, USA

This manuscript has been authored by UT-Battelle, LLC under Contract No. DE-AC05-000R22725 with the U.S. Department of Energy. The United States Government retains and the publisher, by accepting the article for publication, acknowledges that the United States Government retains a non-exclusive, paid-up, irrevocable, world-wide license to publish or reproduce the published form of this manuscript, or allow others to do so, for United States Government purposes. The Department of Energy will provide public access to these results of federally sponsored research in accordance with the DOE Public Access Plan(http://energy.gov/downloads/doe-public-access-plan).

* Correspondence and requests for materials should be addressed to H.B. (email: beih@ornl.gov) 


\title{
Weldability of a High Entropy CrMnFeCoNi Alloy
}

\author{
Z. Wu, S. A. David, Z. Feng, H. Bei * \\ Materials Science and Technology Division, Oak Ridge National Laboratory, Oak Ridge, TN 37831, \\ USA
}

\begin{abstract}
High-entropy alloys are unique alloys in which five or more elements are presented all in high concentrations. To determine its potential as a structural alloy, a model face-centered-cubic $\mathrm{CrMnFeCoNi}$ alloy was selected to investigate its weldability. Welds produced by electron beam welding show no cracking. The grain structures within the fusion zone (FZ) are controlled by the solidification behavior of the weld pool. The weldment possesses mechanical properties comparable to those of the base metal (BM) at both room and cryogenic temperatures. Compared with the BM, deformation twinning was more pronounced in the FZ of the tested alloy.
\end{abstract}

Keywords: High entropy alloy; Weldability; Twinning; Microstructure

* Correspondence and requests for materials should be addressed to H.B. (email: beih@ornl.gov) 
High-entropy alloys (HEAs) are a unique class of alloys in which five or more elemental components are present in equiatomic or near-equiatomic composition [1-3]. These alloys derive their properties not from a single dominant constituent-such as iron in steels, nickel in superalloys, and aluminum in aluminum alloys - but rather from multiple principal elements with the potential for unique combinations of mechanical/physical properties compared with traditional alloys [4]. Some single-phase HEAs, such as the alloys made from equal amount of chromium, manganese, iron, cobalt, and nickel, have unusual and remarkable mechanical properties [1-10]. Equiatomic CrMnFeCoNi has a face-centered-cubic (FCC) crystal structure [8] and exhibits interesting temperature-dependent mechanical behavior [7, 9], showing a strong increase in both yield strength and ultimate tensile strength (UTS) with decreasing temperature, accompanied by a large increase in ductility. The This alloy also displays a high fracture toughness values (exceeding $200 \mathrm{MPa} \mathrm{m}^{1 / 2}$ ) at cryogenic temperature, which are is associated with high tensile strength and ductility [10]. Extensive research has been conducted to understand the microstructure and the deformation mechanisms of CrMnFeCoNi [11-14]. The excellent combination of mechanical properties was attributed to a transition from planar-slip dislocation activity at room temperature to deformation by mechanical nano-twinning with decreasing temperature, which results in continuous steady strain hardening.

The remarkable fracture toughness, comparable to that of the very best cryogenic steelsspecifically certain austenitic stainless steels and high-nickel steels [15-22], which also have outstanding combinations of strength and ductility-puts this alloy among the most damagetolerant materials in that temperature range, making it an ideal material for low-temperature structural applications [10]. For a new HEA to be used for structural applications, it is critical to 
investigate the behavior of the alloy during processing and fabrication. Welding is a critical fabrication technology used in a wide variety of industries, such as energy, aerospace, and ship building. The behavior of a new alloy during welding will remain a key technology issue. $\mathrm{CrMnFeCoNi}$ alloy is no exception, and the behavior of the alloy when exposed to a welding thermal cycle needs to be explored and understood. The capability of being welded without problems arising will enhance the potential of the alloy as an engineering material for structural applications. The primary objective of current work is to investigate the weldability, which was defined by David as "a measure of the ease with which a metal or an alloy can be welded or joined without degradation that is detrimental to the weldment microstructure or properties during or after welding and for the duration of intended service [23]," of CrMnFeCoNi alloy by means of mircrostructural and mechanical property analyses.

Single-phase FCC equiatomic $\mathrm{CrMnFeCoNi}$ was produced by arc-melting the constituent elements (>99.9\% pure) in a water-cooled copper hearth under an argon atmosphere. Similar to the process described previously [24], $12.7 \times 25.4 \times 127 \mathrm{~mm}$ alloy ingots were fabricated by drop casting, followed by homogenization (at $1200^{\circ} \mathrm{C}$ for 24 hours) and water quenching to promote chemical homogeneity. Thermomechanical processing, including longitudinal cold-rolling (to $86 \%$ thickness reduction) and annealing (at $900^{\circ} \mathrm{C}$ for 1 hour), was conducted to obtain equiaxed-grained recrystallization microstructures. As shown in Fig. 1(a), annealing twins are visible in many grains, and the thickness of the annealing twin is on the tens of micrometers scale. Using a linear intercept method, the average grain size of the alloy (twin boundaries are not counted in the calculation) was determined to be $\sim 20 \mu \mathrm{m}$.

To evaluate the weldability of an alloy, ideally, a testing procedure that duplicates the conditions the materials experiences during weld fabrication should be employed. In most cases, 
it is not possible to demonstrate weldability using a full-size mockup. Also, in most of the alloy design and screening stages, the dimensions and amounts of experimental materials available are limited. Therefore, initial weldability tests are conducted with thin sheets of alloys produced on a laboratory scale, using autogenous welding or melt run experiments. This is a quick way to assess the response of the material to a weld thermal cycle; in particular, the susceptibility to thermal cracking during welding can be determined. Similar to the laser cladding process $[25$, 26], materials might form non-equilibrium microstructures due to the large cooling rate during melting and solidification cycles, although laser cladding is normally used to add new materials (with different composition) to the substrate, which is different from the electron beam welding process used in this study. Here, several autogenous welds were made on thin sheets $(\sim 1.5 \mathrm{~mm}$ thick) of the alloy using an electron beam welder. The power level used was $125 \mathrm{kV}, 5 \mathrm{~mA}$ at a welding speed of $9.53 \mathrm{~mm} / \mathrm{s}$.

During welding, the heat source destroys the original microstructure of the material by melting it, creating a fusion pool (FZ)) that solidifies upon cooling. Beside the fusion zone is the heat affected zone (HAZ), a region of the metal that experiences heat but does not melt. The rest of the material, which is not affected by the welding heat, is the base metal (BM). Figure 1(a) shows the interaction of the heat source with the material. The microstructure of both the top surface and the transverse sections of the weld were observed using backscattered (BSE) scanning electron microscopy (SEM) and electron backscatter diffraction (EBSD), as shown in Fig. 1(b-e). Neither solidification cracking nor cracks in or near the HAZ were observed, indicating the promising weldability of this HEA. The cracking susceptibility of an alloy depends on its composition, its segregation behavior, and the microstructural characteristics (e.g., formation of intermetallic phases) within the material [27-29]. Solidification cracking often 
occurs during the welding of an alloy that has a wide freezing range and the presence of lowmelting-point segregates along grain boundaries; these can increase the crack susceptibility of the boundaries when they are under tension stress due to thermal contraction and/or solidification shrinkage. Equiatomic $\mathrm{CrMnFeCoNi}$ has a narrow freezing range of $\sim 60{ }^{\circ} \mathrm{C}[30]$ and large high temperature solid solubility between most pairs of two elements (e.g., complete solid solutions between $\mathrm{Ni}$ and $\mathrm{Co}$ ); therefore the possibility of hot cracking susceptibility and compositional segregation, still depending on the solidification rate, is expected to be low. Fig. 1 (f) is an elemental mapping using energy-dispersive spectroscopy (EDS) across the FZ, revealing a uniform distribution of all five individual elements. Compared with the BMs, which have fine equiaxed grains, the $\mathrm{FZ}$ of the $\mathrm{CrMnFeCoNi}$ weld revealed a relative coarse and elongated grain structure.

The microstructural development of the FZ depends to a large extent on the solidification behavior of the weld pool [23, 31-34]. The principles of solidification control the size and shape of the grains, the segregation of the elements, and other defects. The initial growth of solids in the FZ occurs epitaxially from the partially melted grains at the fusion line [31]. In general, during solidification, the grains tend to grow in the direction perpendicular to the solid/liquid interface, since this is the direction of the maximum temperature gradient and thus the maximum driving force for solidification. However, grains also have their own preferred direction of growth, called the easy growth direction; e.g., $<100>$ is the easy-growth direction for both FCC and body-centered-cubic materials. Therefore, the initial growth of the partially melted grains in the base metal is followed by a competitive growth process during welding. Grains with their easy growth direction parallel to the direction of the maximum temperature gradient will grow 
more easily and crowd out other grains of which the easy growth direction deviates significantly from the direction of the maximum temperature gradient.

Weld pool shape is also important in the development of grain structure and the grain selection process [23, 27, 31]. For welds made at low or moderate welding speed, particularly in thin materials, the weld pool assumes an elliptical shape (Fig. 2). As the welding speed increases, the pool shape changes to a teardrop shape. For an elliptically shaped weld pool, the optimum conditions for grains to grow are such that no grain from the fusion line experiences preferred growth for an extended period of time. Therefore, many grains from the fusion line reach the centerline of the FZ, resulting in a fine grain structure in the FZ. In contrast, for a teardropshaped pool, the thermal and crystallographic conditions are such that only a few favorably oriented grains grow at the expense of less favorably oriented grains, resulting in coarse structures in the FZ as is the case in the current study. Similar observations were made for fusion welding of FCC iridium [31, 32] and austenitic stainless steel [35].

In most traditional materials, the significant growth of grains in the FZ could have severe detrimental effects on the mechanical properties, i.e., a significant loss of ductility and/or strength in the weld due to low-melting-point segregations along the grain boundaries [27]. The coarse grain structures in the FZ could also reduce the capability of the grains to accommodate plastic deformation and thus further decrease ductility. To investigate the mechanical properties of $\mathrm{CrMnFeCoNi}$ after welding, flat dog-bone-shaped, cross-weld specimens with a gauge length of $10 \mathrm{~mm}$ were cut from the welded sheets with their tensile axes perpendicular to the welding direction. Nine Vickers microhardness indents spaced $1 \mathrm{~mm}$ apart were made along the specimen gauge lengths to measure the uniform elongation to fracture and to calibrate the engineering stress vs. engineering strain curves from the tensile load-displacement data. Tensile tests were 
performed with a screw-driven Instron machine at an engineering strain rate of $10^{-3} \mathrm{~s}^{-1}$ at temperatures of 77 and $293 \mathrm{~K}$. Three individual tests were conducted at each temperature, showing good reproducibility.

Figure 3 shows the representative engineering stress vs. engineering strain curves of the welds tested at two temperatures, 77 and $293 \mathrm{~K}$. The $0.2 \%$ offset yield strengths $\left(\sigma_{\mathrm{y}}\right)$, ultimate tensile strengths $\left(\sigma_{U T S}\right)$, and uniform elongations to fracture $\left(\mathrm{e}_{\mathrm{f}}\right)$ are labeled in the figure. It can be seen that, although it has a coarse grain microstructure, the welded CrMnFeCoNi alloy has tensile strength and ductility comparable to that of the base material, which have been extensively measured previously. The tensile strength and ductility increased significantly when the deformation temperature decreased from 293 to $77 \mathrm{~K}$.

$\mathrm{CrMnFeCoNi}$ has been shown to be an FCC solid solution with a low stacking fault energy of $20-25 \mathrm{~mJ} \mathrm{~m}^{-2}$ [36]; therefore, twinning is expected to act as an additional deformation mechanism in low-temperature (e.g., $77 \mathrm{~K}$ ) deformation and to provide strengthening because of the additional internal twin boundaries generated. The increased tensile strength and ductility at low temperatures could also be caused by the twinning effect. A few studies of the BMs have also supported the twinning effect in this alloy $[7,10]$. To investigate whether the persistence of the BM tensile strength and ductility of the weld is related to the twinning effect, the microstructure of the weld metal was characterized after tensile fracture. Both the BM region and the FZ were imaged. It can be seen from Fig. 4 that the highly distorted grains, with significant in-grain orientation fluctuations after fracture, mainly exhibit orientations with their $\left\langle\begin{array}{lll}1 & 1 & 1\end{array}\right\rangle$ crystallographic direction parallel to the tensile axis. Under tension conditions, the $\left\langle\begin{array}{lll}1 & 1 & 1\end{array}\right\rangle$ //tensile axis grains have been reported [37-40] to be more prone to twinning because of the fact that the Schmid factor for the mechanical twinning $(0.31)$ is larger than that for dislocation slip 
(0.27). In the FZ, under fracture at both temperatures, the microstructures were dominated by a tremendous number of band-like features of varying thickness and spacing. Only a few parts of the microstructure were free of these bands. For better visualization of these bands, the selected area was magnified and a slow scan with a step size of $20 \mathrm{~nm}$ was conducted. A corresponding high-magnification Kikuchi pattern quality (KPQ) map composed of numerous straight dark lines in a micron-scale band (indicated with a red line) is shown in Fig. 4(c). To identify details of the bands, misorientations along the red line were measured and the results were plotted in Fig. 4(d). The image shows that whenever the scan passed the lines, there was a $60^{\circ}$ misorientation, indicating that these bands are composed of $\Sigma 3$ mechanical twins.

The KPQ map in Fig. 4 (c) also shows that the thickness of each individual twin is at the nanoscale. The bands are bundles of these nanoscale twins, which often nucleate at the grain boundaries at the early deformation stage, owing to the tendency for dislocation accumulation along the boundaries, and increase in number as the strain progresses. The zigzag-like microstructure shown in Fig. 4 is formed when more and more twins bundle together and the secondary twinning systems are activated. Twinning can be considered a nucleation and growth process; in pure metals and alloys, it is initiated by preexisting dislocations that dissociate into multi-layered stacking faults, creating a twin nucleus. The nucleation of deformation twins proceeds by specific partial dislocation reactions, and it has a critical stress that must be reached. Grain boundaries are the main sites of twin nucleation in single-phase solid solutions because of the ease of stress concentration at the boundaries. Fine-grained materials have more grain boundaries and thus more nucleation sites for twinning; however, under deformation, dislocations in the fine-grained materials tend to have a fairly homogeneous distribution and to form dislocation cell structures. These cell-forming crystals do not twin upon deformation. In 
coarse-grained materials, high-density dislocation substructures, such as high-density dislocation walls, tend to form; and it is easier for the critical stresses for twin initiation to be reached. Thus, although the coarse grain structure does not easily accommodate plastic deformation, it could favor the formation of mechanical twinning [41-43]. A comparison of the post-fracture microstructures between the FZ [Fig. 4 (a, b)] and the BM regions [Fig. 4 (e, f)] supports this hypothesis. Under both temperatures, the densities of the twin bands are much higher in the FZ than in the BM region; this can be more clearly observed in the sample tested at $293 \mathrm{~K}$, where twinning occurred even more rarely in the BM region. The grain-size-dependence of twinning activities in this alloy is an interesting subject for future studies.

The deformation twinning enhances strain hardening and postpones necking. Consistently, the representative fracture surfaces in Fig. 3(b) show that there is only minor macroscopic necking in the weld. Microscopically, the fracture surface exhibits ductile dimples on the fracture surfaces. The fracturing occurs as a result of the nucleation of microvoids. Some particles were found inside the voids of the fracture surface; they act as initiation sites for void formation. It was indicated [9] that the particles are likely oxides associated with manganese. The coarse grain structure in the FZ normally makes accommodating plastic deformation difficult. However, it may be compensated by the significant amounts of twinning activity, causing the weld to maintain the strength and ductility of the BMs.

In summary, the weldability of $\mathrm{CrMnFeCoNi} \mathrm{HEA}$ was tested using autogenous thin sheet welding experiments. After welding, no solidification cracking was observed; moreover, the welded materials maintained the strength and ductility of the BMs at both room and cryogenic temperatures, indicating good weldability of the HEA in this condition. Compared with the BM, more pronounced deformation twinning was observed in the $\mathrm{FZ}$ of the tested alloy. 


\section{Acknowledgments}

This work was supported by the U.S. Department of Energy, Office of Science, Basic Energy Science, Materials Science and Engineering Division. 


\section{Reference:}

[1] Yeh JW, Chen SK, Lin SJ, et al. Adv. Eng. Mater 2004; 6: 299.

[2] Bei H. US Patent 09150945.

[3] Pickering EJ, Jones NG. Inter. Mater. Rev 2016; 61(3): 183.

[4] Jin K, Sales BC, Stocks GM, et al. Sci. Rep. 2016; 6, 20159.

[5] Senkov ON, Wilks GB, Miracle DB, et al. Intermetallics 2010; 18: 1758.

[6] Senkov ON, Wilks GB, Scott JM, et al. Intermetallics 2011; 19:698.

[7] Otto F, Dlouhy A, Somsen Ch, et al. Acta. Mater 2013; 61: 5743.

[8] Cantor B, Chang ITH, Knight P, et al. Mater. Sci. Eng A 2004; 375-377: 213.

[9] Wu Z, Bei H, Pharr GM, et al. Acta Mater 2014; 81:428.

[10] Gludovatz B, Hohenwarter A, Catoor D, et al. Science 2014; 345: 1153.

[11] Stepanov ND, Shaysultanov DG, Yurchenko NY, et al. Mater Sci Eng A 2015; 636: 188.

[12] Woo W, Huang EW, Yeh JW, et al. Intermetallics 2015; 62: 1.

[13] Wu Y, Liu WH, Wang XL, et al. App Phys Lett 2014; 104: 051910.

[14] Wu Z, Parish CM, Bei H. Journal of Alloys and Compounds 2015; 647: 815.

[15] Mills WJ. Int. Mater. Rev 1997; 42: 45.

[16] Sokolov M, et al., in Effects of Radiation on Materials: $20^{\text {th }}$ International Symposium, S. Rosinski, M. Grossbeck, T. Allen, A. Kumar, Eds. (ASTM International, West Conshohocken, PA, 2001), pp. 125-147.

[17] Strife JR, Passoja DE. Metall. Trans. A 1980; 11: 1341.

[18] Syn CK, Morris JW, Jin S. Metall. Trans. A 1976; 7: 1827.

[19] Read DT, Reed RP. Cryogenics 1981; 21: 415.

[20] Stout RD, Wiersma SJ. in Advances in Cryogenic Engineering Materials, R. P. Reed, A. F. Clark, Eds. (Springer, New York, 1986), pp. 389-395.

[21] Shindo Y, Horiguchi K. Sci. Technol. Adv. Mater 2003; 4: 319-326.

[22] Sa JW, et al., in Twenty-First IEEE/NPS Symposium on Fusion Engineering 2005 (IEEE, Piscataway, NJ, 2005), pp. 1-4.

[23] David SA, Siefert JA, Feng Z. Sci. Tech. of Weld. Joining 2013; 18(8): 631.

[24] Wu Z, Bei H, Otto F, et al. Intermetallics 2014; 46: 131.

[25] Ocelik V, Janssen N, Smith SN, et al.. JOM 2016; DOI: 10.1007/s11837-016-1888-z. 
[26] Zhang H, Pan Y, He Y. Mater. Des 2011, 32(4): 1910.

[27] Kou S. Welding metallurgy 1987 ,

[28] Hondros ED, Seah MP. Int. Metals. Rev 1977; 222: 12.

[29] Kah DH, Dickinson DW. Weld J 1981; 60: 135s.

[30] Gali A, George EP. Intermetallics 2013; 39:74

[31] David SA, Vitek JM. Int. Mater. Rev 1989; 34(1): 213.

[32] David SA, Liu CT. Mater. Techno 1980; 7(1): 102.

[33] David SA, DebRoy T. Science 1992; 257: 497.

[34] David SA, DebRoy T, Vitek JM. MRS Bulletin 1994; XIX: 29

[35] Viliafuerte JC, Kerr HW. Metall. Trans. A 1990; 21: 979.

[36] Zaddach AJ, Niu C, Koch CC et al.. JOM 2013; 65: 1780.

[37] Lee YK. Scr Mater 2012; 66: 1002.

[38] Goodchild D, Roberts WT, Wilson DV. Acta Metall 1970; $18: 1137$.

[39] Kestenbach HJ. Phil Mag 1977; 36: 1509.

[40] Idrissi H, Ryelandt L, Veron M, et al.. Scr Mater 2009; 60: 941.

[41] Christian JW, Mahajan S. Prog. Mater. Sci 1995; 39: 1.

[42] Remy L. Acta Metall 1977; 25: 711.

[43] Vercammen S, Blanpain B, De Cooman BC, et al.. Acta Mater 2004; 52: 2005. 


\section{Figure Captions}

Fig. 1 Backscattered electron images of (a) initial alloys, (b) the top surface, and (c) the transverse surface of the weld. (d, e) EBSD images of the top surface and the transverse surface of the weld, respectively. (f) Elemental distribution around the weld.

Fig. 2 (a) Schematic diagram showing the three zones within a weldment; (b) and (c) show comparisons of the weld pool shapes. Adapted from ref [31].

Fig. 3 (a) Tensile engineering stress-strain curves of the welded CrMnFeCoNi alloy deformed at 77 and $293 \mathrm{~K}$. (b) The fracture surfaces of the weld tensile deformed to fracture at $77 \mathrm{~K}$

Fig. 4 (a, b) Microstructures of the fusion zone and $(e, f)$ base metal region after tensile deformation to fracture at 77 and $293 \mathrm{~K}$. Color represents inverse pole figure map projected on tensile direction (horizontal). (c) High-magnification Kikuchi pattern quality map of the marked area and (d) corresponding misorientation as a function of distance for the red line across the individual bands. 


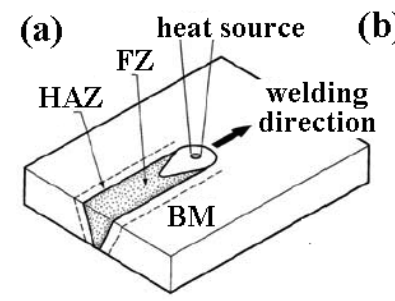

(b)

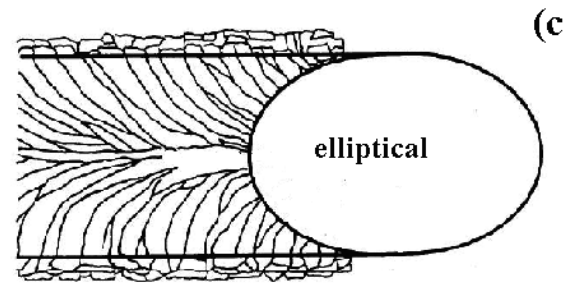

(c)

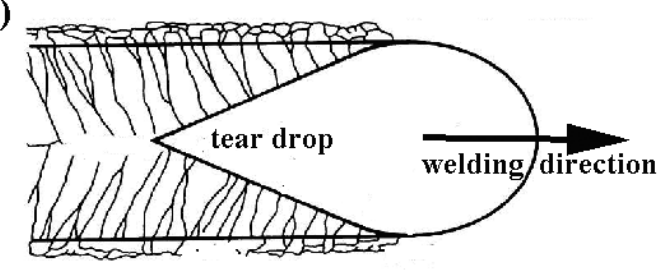

Figure 1 


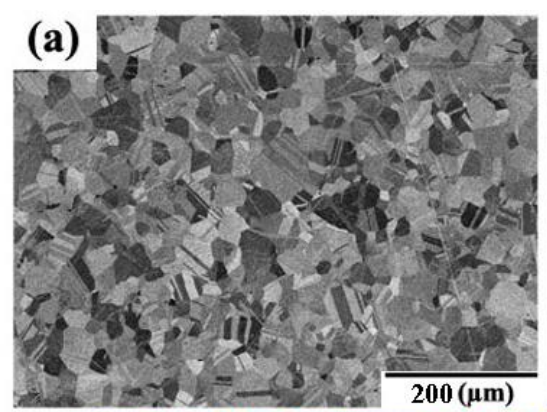

(d)
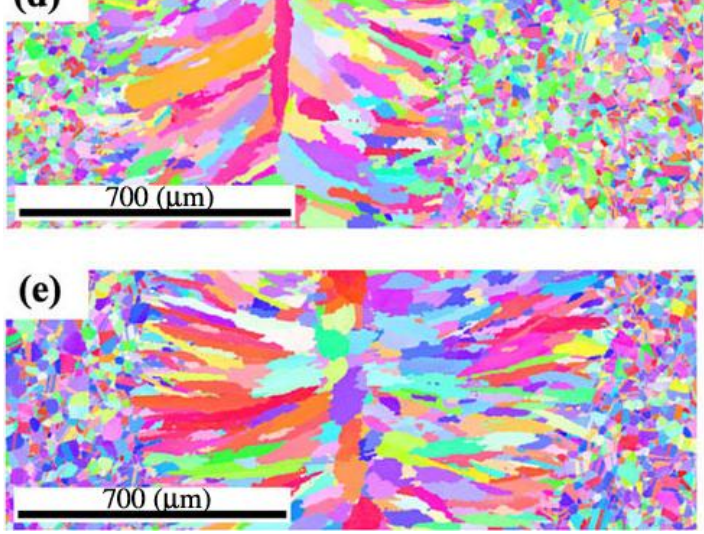

(b)

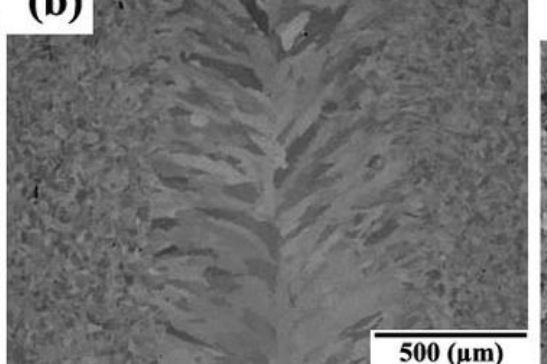

(c)
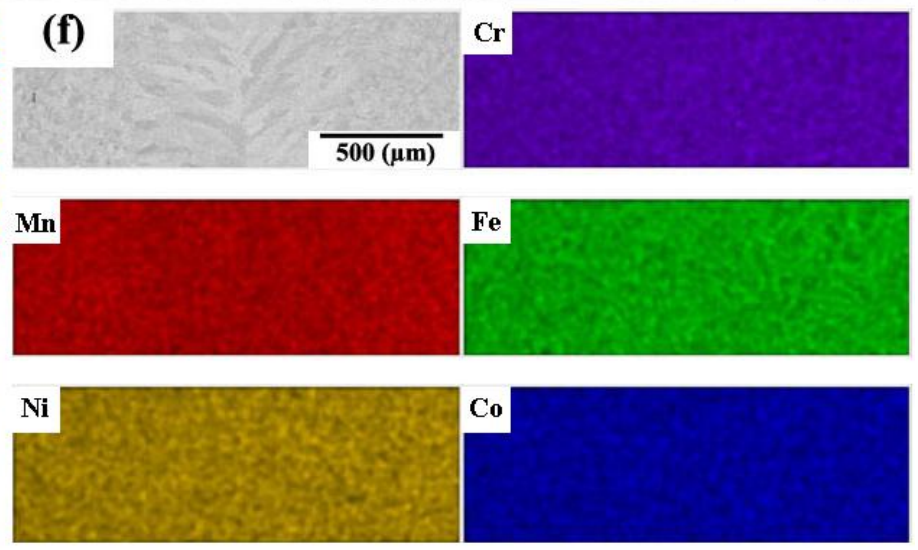

Figure 2 


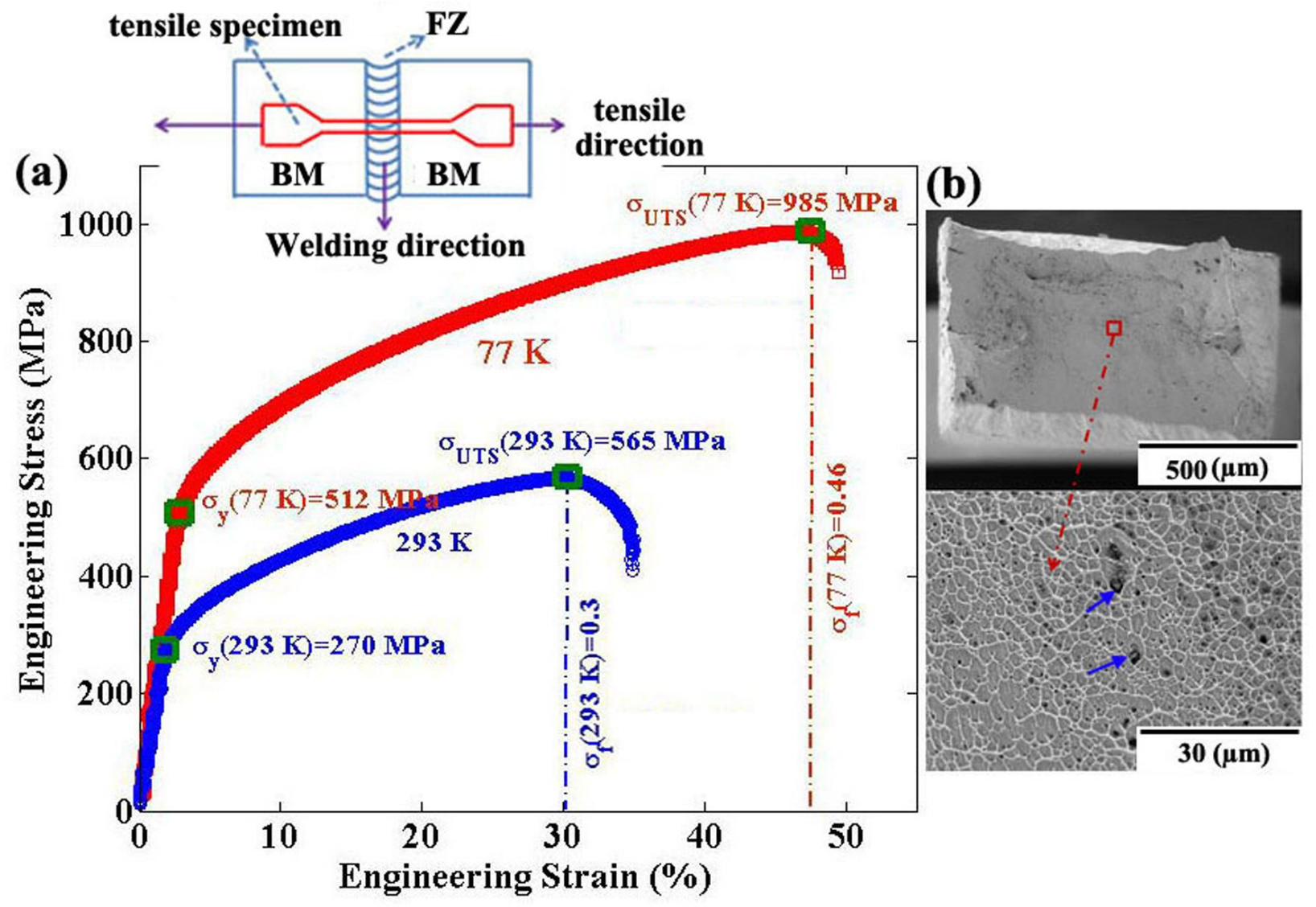

Figure 3 


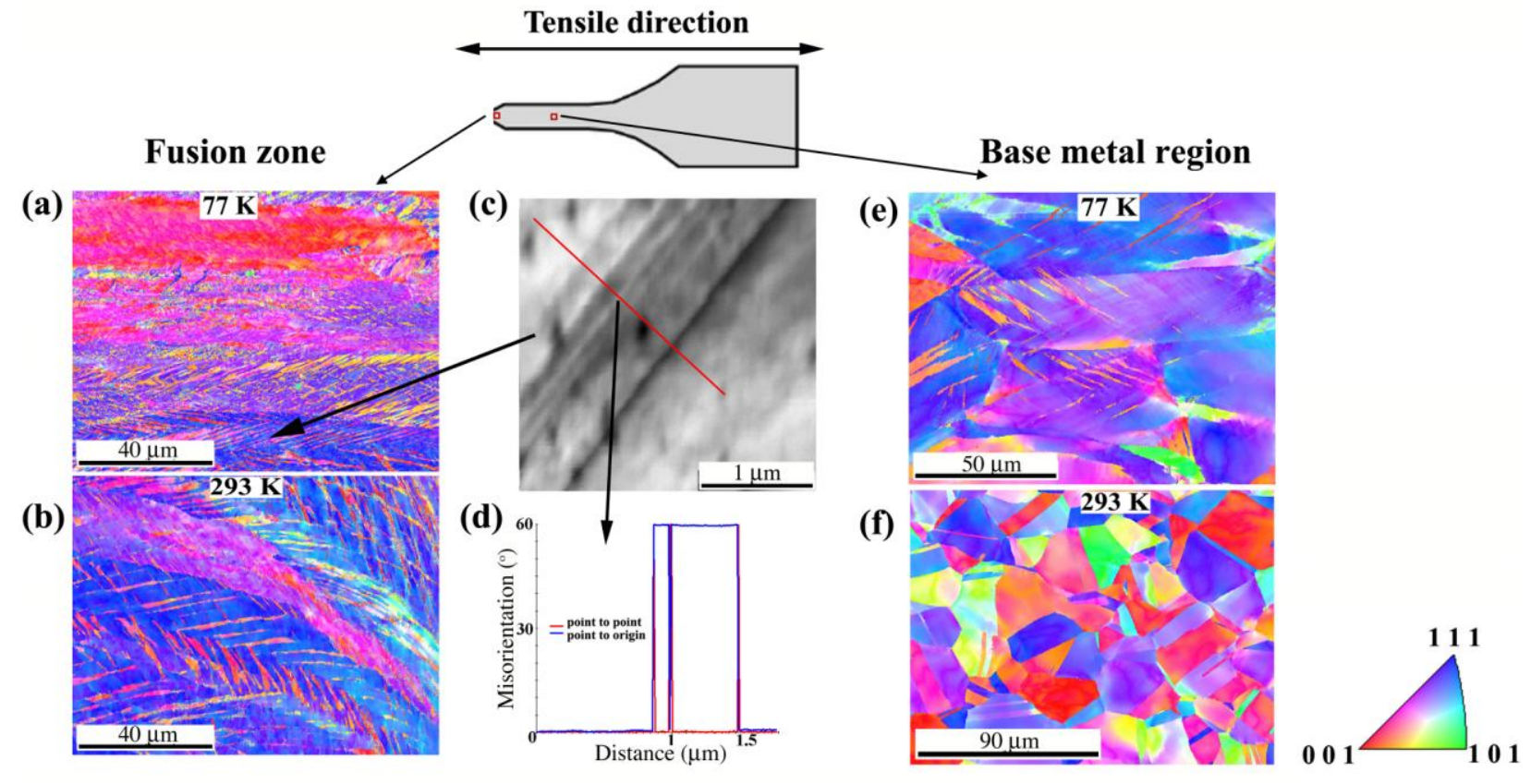

Figure 4 


\section{Graphical Abstract}

\section{Weldability of a High Entropy CrMnFeCoNi Alloy}
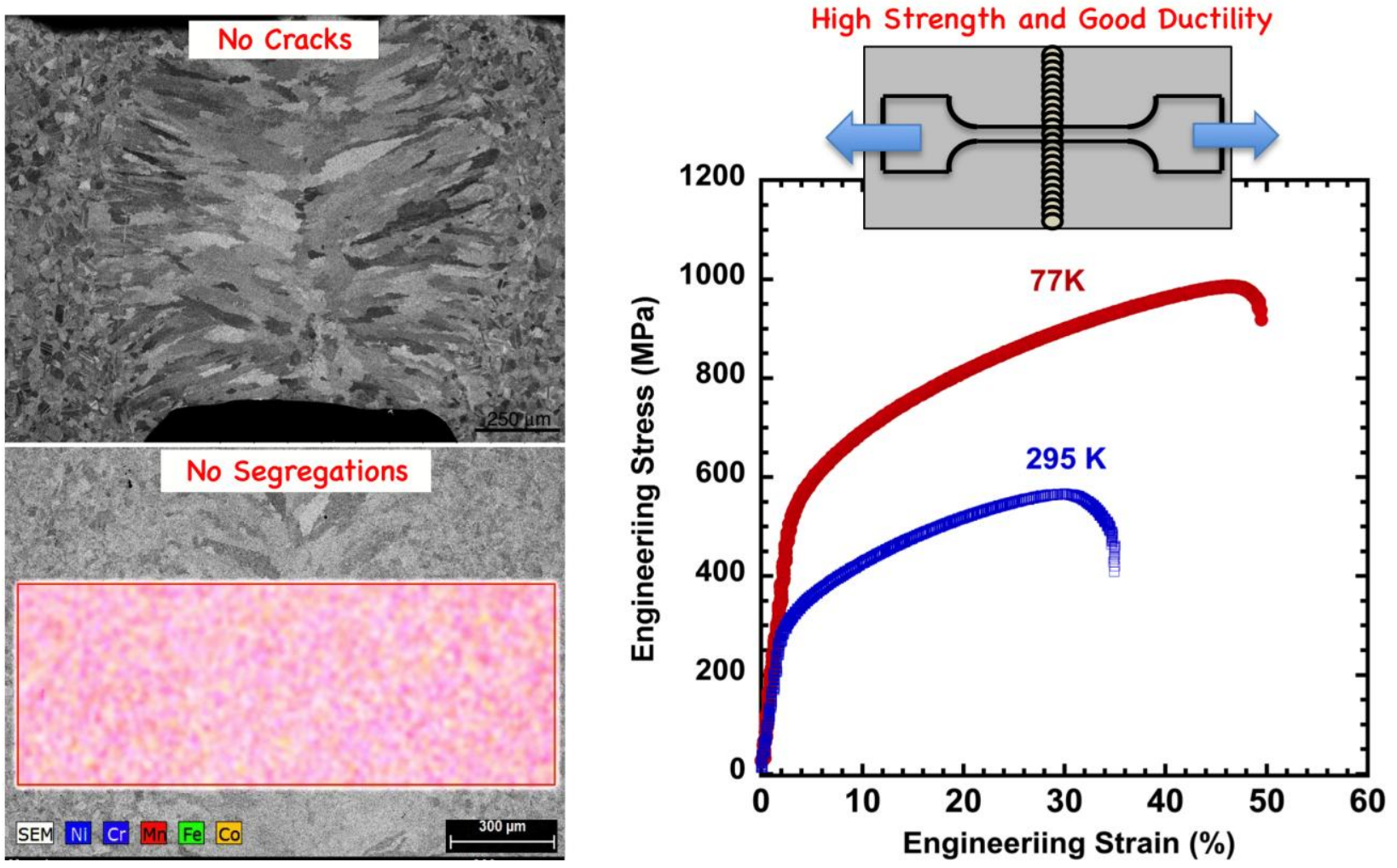